\title{
Triplet repeats in clinical subtypes of schizophrenia: variation at the DRPLA (B 37 CAG repeat) locus is not associated with periodic catatonia
}

\author{
Short Communication
}

\author{
K. P. Lesch ${ }^{1}$, G. Stöber ${ }^{1}$, U. Balling ${ }^{1}$, E. Franzek ${ }^{1}$, S. H. Li ${ }^{2}$, C. A. Ross ${ }^{2}$, M. Newman ${ }^{3}$, \\ H. Beckmann' ${ }^{1}$, and P. Riederer ${ }^{1}$ \\ ${ }^{1}$ Department of Psychiatry, University of Würzburg, Würzburg, \\ Federal Republic of Germany \\ ${ }^{2}$ Laboratory of Molecular Neurobiology, Departments of Psychiatry and Neuroscience, \\ Johns Hopkins University, School of Medicine, Baltimore, MD, U.S.A. \\ ${ }^{3}$ Biological Psychiatry Laboratory, Department of Psychiatry, \\ Hadassah-Hebrew University Medical Center, Jerusalem, Israel
}

Accepted September 8, 1994

Summary. Clinical evidence for a dominant mode of inheritance and anticipation in periodic catatonia, a distinct subtype of schizophrenia, indicates that genes with triplet repeat expansions or other unstable repetitive elements affecting gene expression may be involved in the etiology of this disorder. Because patients affected with dentatorubral-pallidoluysian atrophy (DRPLA) may present with "schizophrenic" symptoms, we have investigated the DRPLA (B 37 CAG repeat) locus on chromosome 12 in 41 patients with periodic catatonia. The B 37 CAG repeat locus was highly polymorphic but all alleles in both the patient and control group had repeat sizes within the normal range. We conclude that variation at the DRPLA locus is unlikely to be associated with periodic catatonia. The evidence for dominant inheritance and anticipation as well as the high prevalence of human brain genes containing trinucleotide repeats justifies further screening for triplet repeat expansions in periodic catatonia.

Keywords: Association study, B 37 CAG repeat locus, chromosome 12, schizophrenia, periodic catatonia.

\section{Introduction}

Genes containing repetitive DNA elements, such as triplet repeats, may be expanded, unstable and a potential cause for some psychiatric disorders (Ross 
et al., 1993). Triplet repeats may therefore be the molecular correlate of the clinical phenomenon of anticipation observed in subtypes of schizophrenia. Anticipation describes the unusual pattern of inheritance in genetic disorder whose severity increase and age of onset decrease in successive generations. This phenomenon, however, tends to be challenged as an assessment bias (Harper et al., 1992). The recent discovery of a new form of human mutation provided a specific biological explanation in several inherited neurological and neuropsychiatric diseases with anticipation (Mandel, 1993). In Huntington's disease, myotonic dystrophy, fragile $\mathrm{X}$ syndrome, spinocerebellar ataxia type 1 , dentatorubral-pallidoluysian atrophy (DRPLA) (Koide et al., 1994; Nagafuchi et al., 1994), unstable expansion of trinucleotide repeats were identified in coding/non-coding regions of distinct genes. Repeat length and instability is directly associated with increased severity and earlier age of onset in successive generations.

Recently, an investigation of familial aggregation of psychoses in pedigrees with schizophrenic disorders strongly suggested a dominant mode of inheritance in periodic catatonia, a distinct subtype of schizophrenia (Stöber et al., 1994). As compared to other forms of catatonic schizophrenia (age-correlated morbidity risk, $4.6 \%$ ) with a chronic progressive course and irreversible, well-defined defective states (Franzek and Beckmann, 1992), periodic catatonia was characterized by an age-corrected morbidity risk with homogenous psychoses of $26.9 \%$. Moreover, a pairwise comparison of patients and their parents revealed patterns of genetic anticipation. The course of periodic catatonia is bipolar in both hyperkinetic as well as akinetic states. Typically, symptoms of one pole are combined with those of the other pole. The distortion of psychomotor activity leads to grimaces, parakinetic movements, stereotypies, impulsive actions with aggressiveness, and negativistic behavior. Although remissions occur after acute episodes, residual states of varying degrees finally develop with increasing poverty of movements, blunted affect, and lack of motivation.

The clinical evidence for a dominant mode of inheritance and anticipation in periodic catatonia indicates that genes with triplet repeat expansions or other unstable repetitive elements affecting gene expression may be involved in its etiology. Because patients affected with DRPLA may also present with "schizophrenic" symptoms (Koide et al., 1994), such as delusion and hallucination, variation at the DRPLA (B 37 CAG repeat) locus on chromosome 12 was investigated in periodic catatonia.

\section{Subjects and methods}

\section{Recruitment of patients and diagnostic assessment}

Fourty-one unrelated German caucasians (19 male, 22 female) meeting the DSM-IIIR criteria for schizophrenia, catatonic subtype (APA 1987), and the criteria for periodic catatonia (Leonhard, 1979, 1980) were recruited from inpatients at the Department of Psychiatry, University of Würzburg, and from wards with chronically ill patients at the Lohr/Main Psychiatric State Hospital. The patients with periodic catatonia included in this 
study represent a subsample of a study on familial aggregation of psychoses in pedigrees with schizophrenic disorders published previously (Stöber et al., 1994). After an initial screening of the hospital records by G. S., patients, who fulfilled the criteria for catatonic schizophrenia, were diagnosed along the lines of Leonhard's nosology by personal examinations by two independently working experienced clinical psychiatrists (E. F. and H. B.) on the basis of extensive case notes which did not contain information on familial affliction (Cohen's Kappa, 0.93). The mean age $[ \pm \mathrm{SD}]$ of the patients with periodic catatonia was $50.7 \pm 14.4$ years, the duration of illness was $28.3 \pm 13.7$ years, and the age of initial hospitalization was $23.2 \pm 7.3$ years. Thirty unrelated German caucasian ( 10 males, 20 females; mean age $25.1 \pm 3.9$ years) were included as controls. The study was approved by the local ethics committee.

\section{$P C R$ analysis of the $C A G B 37$ repeat}

High molecular weight genomic DNA was extracted from EDTA-anticoagulated peripheral blood according to routine procedures. The $\mathrm{B} 37 \mathrm{CAG}$ repeat was amplified from genomic DNA ( 50 ng) by PCR ( $30 \mathrm{~s}$ at $95^{\circ} \mathrm{C}, 30 \mathrm{~s}$ at $64^{\circ} \mathrm{C}, 1 \mathrm{~min}$ at $72^{\circ} \mathrm{C}$ for 30 cycles) with the amplimers B 37-1 (5'-CACCAGTCTCAACACATCACCATC- $\left.3^{\prime}\right)$ and B 37-2 (5'CTCCAGTGGGTGGGGAAATGC-3'). The PCR products were analyzed on a $2 \%$ agarose gel. For accurate determination of the number of CAG repeat units, PCR was performed with $\sim 50 \mathrm{KBq}$ of $\left[\alpha_{-}{ }^{32} \mathrm{P}\right] \mathrm{dATP}$ and the size of the product estimated in comparison with a standard sequencing ladder by $6 \%$ denaturing polyacrylamide gel electrophoresis.

\section{Results}

The distribution of B 36 CAG repeat alleles in patients with periodic catatonia and controls is depicted in Fig. 1. Eighty-two chromosomes were analyzed in the patient sample and 40 in the controls. The B 37 CAG repeat locus is highly polymorphic (17 alleles) but all alleles in both the patient and control group had repeat sizes within the previously reported distribution in normal subjects (Koide et al., 1994; Nagafuchi et al., 1994). The heterozygosity of $81 \%$ in the

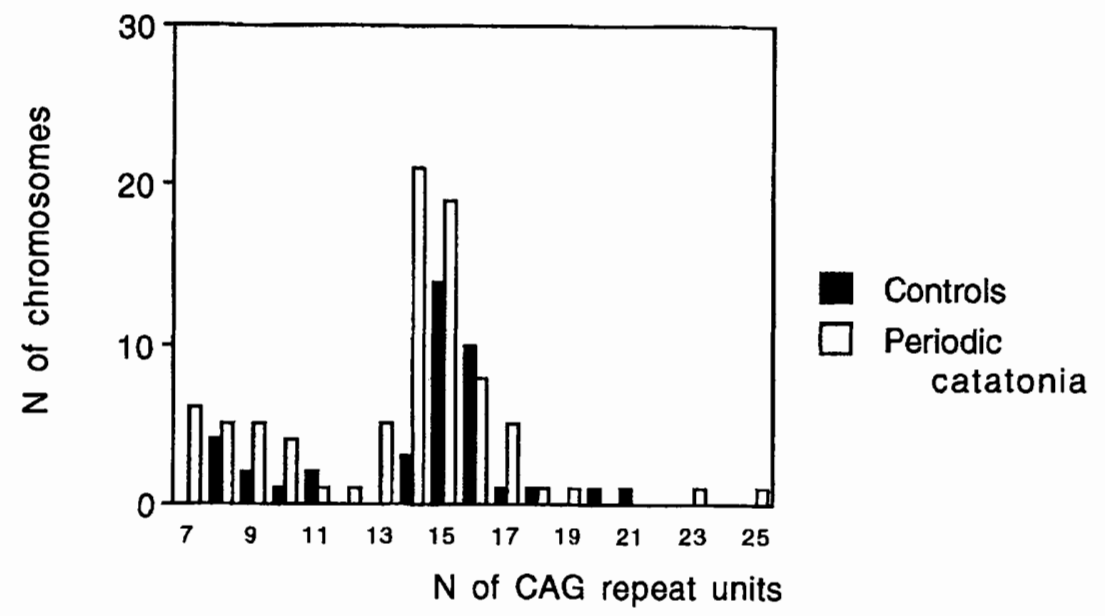

Fig. 1. Distribution of B 37 CAG repeat alleles in patients with periodic catatonia and controls 
patient group and of $85 \%$ in the controls was not significantly different to that observed in a normal population. The modal repeat length was 14 repeats in the patients and 15 repeats in the control group, which corresponds to an allele size of 42 and 45 base pairs, respectively. There was no significant difference in the mean allele size between the patient and the control sample (mean $\pm \mathrm{SD}$, $40.3 \pm 10.5$ vs $42.8 \pm 9.5$ bas paris; $\mathrm{t}=1.24$, $\mathrm{df}=120, \mathrm{p}>0.22$, unpaired $\mathrm{t}$ test).

\section{Discussion}

Because the etiology of schizophrenia is likely to be heterogenous and the strength of the genetic contribution to schizophrenia may vary considerably among its subtypes, we have screened the DRPLA (B 37 CAG repeat) locus in a subtype of schizophrenia with strong evidence for a dominant pattern of inheritance and anticipation. The B 37 CAG repeat locus was highly polymorphic but all alleles in both the patient and control group had repeat sizes within the normal range. Our results further support and complement the findings of a recent study by Rubinsztein et al. (1994), who also failed to detect abnormalities in the DRPLA (B 37 CAG repeat) locus in an unselected sample of 55 patients with a schizophrenic disorder.

An increased familial loading in catatonic psychoses compared to paranoid or hebephrenic schizophrenia was detected in earlier studies (Leonhard, 1979; Scharfetter and Nuesperli, 1980). In aggreement with Leonhard's findings (1979), we have previously demonstrated that periodic catatonia exhibits a morbidity risk of $26.9 \%$ among first degree relatives (Stöber et al., 1994). High familial loading and pronounced vertical transmission are consistent with a dominant mode of inheritance. In addition, anticipation was observed in 27 of 29 unilineal transmitted families with periodic catatonia and occured irrespective of the disorder's maternally or paternally derivation. The molecular basis of anticipation, expansion of unstable trinucleotide repeats, was recently discovered in several neurologic and neuropsychiatric disorders, and McInnis et al. (1993) and Ross et al. (1993) noted that anticipation may be observed in bipolar affective disorder and subtypes of schizophrenia. The evidence for anticipation in families with periodic catatonia indicates that genes with triplet repeat expansions or other unstable repetitive elements affecting gene expression may also be involved in the etiology of this subtype of schizophrenia. Although no abnormality was found in the DRPLA (B 37 CAG repeat) locus of patients with periodic catatonia in this initial step of a genome-wide screening project, the high prevalence of human brain genes containing trinucleotide repeats ( $\mathrm{Li}$ et al., 1993) clearly justifies further screening for triplet repeat expansions in periodic catatonia.

In conclusion, variation at the DRPLA locus is unlikely to confer susceptibility to periodic catatonia. Because the high genetic loading, dominant inheritance pattern, and clinical evidence for anticipation implicates genes with triplet repeat expansion or other unstable repetitive elements affecting gene 
expression, periodic catatonia and possibly other subtypes of schizophrenia continue to represent promising candidates for further screening for abnormalities in genes with tri- and oligonucleotide repeats.

\section{References}

American Psychiatric Association (1987) Diagnostic and statistical manual of mental disorders, 3rd ed, revised. APA, Washington DC

Franzek E, Beckmann H (1992) Schizophrenia: not a disease entity? A study of 57 longterm hospitalized chronic schizophrenics. Eur J Psychiatry 6: 97-108

Harper PS, Harley HG, Reardon W, Shaw DJ (1992) Anticipation in myotonic dystrophy: new light on an old problem. Am J Hum Genet 51: 10-16

Koide R, Ikeuchi T, Onodera O, Tanaka H, Igarashi S, Endo K, Takahashi H, Kondo R, Ishkawa A, Hayahi T, Saito M, Tomoda A, Miike T, Naito H, Ikuta F, Tsuji S (1994) Unstable expansion of CAG repeat in hereditary dentatorubral-pallidoluysian atrophy (DRPLA). Nature Genetics 6: 9-12

Leonhard K (1979) The classification of endogenous psychoses. Irvington, New York

Leonhard K (1980) Contradictory issues in the origin of schizophrenia. $\mathrm{Br} \mathrm{J}$ Psychiatry 136: $437-444$

Li SH, Mc Innis MG, Margolis RL, Antonarakis SE, Ross CA (1993) Novel triplet repeat containing genes in human brain: cloning, expression, and length polymorphisms. Genomics 16: 572-579

Mandel JL (1993) Questions of expansion. Nature Genet 4: 8-9

McInnis MG, McMahon FJ, Chase GA, Simpson SG, Ross CA, DePaulo JR (1993) Anticipation in bipolar affective disorder. Am J Hum Genet 53: 385-390

Nagafuchi S, Yanagisawa H, Sato K, Shirayama T, Ohsaki E, Bundo M, Takeda T, Tadokoro K, Kondo I, Murayama N, Tanaka Y, Kikushima H, Umino K, Kurosawa H, Furukawa T, Nihei K, Inoue T, Sano A, Komure O, Takahashi M, Yoshizawa T, Kanazawa I, Yamada M (1994) Dentatorubral and pallidoluysian atrophy expansion of an unstable CAG trinucleotide on chromosome 12p. Nature Genetics 6: 14-18

Ross CA, Mc Innis MG, Margolis RL, Li SH (1993) Genes with triplet repeats: candidate mediators of neuropsychiatric disorders. Trends Neurosci 16: 264-272

Rubinsztein DC, Leggo J, Goodburn S, Barton DE, Ferguson-Smith MA, Ross CA, Li SH, Lofthouse R, Crow TJ, Delisi LE (1994) B 37 repeats are normal in most schizophrenic patients (letter). Br J Psychiatry 164: 851-852

Scharfetter C, Nuesperli M (1980) The group of schizophrenias, schizoaffective psychoses, and affective disorders. Schizophr Bull 6: 586-591

Stöber G, Franzek E, Lesch KP, Beckmann H (1994) Periodic catatonia: a schizophrenic subtype with dominant inheritance and anticipation. Eur Arch Psychiatr Clin Neurosci (in press)

Authors' address: K. P. Lesch, M.D., Department of Psychiatry, University of Würzburg, Füchsleinstrasse 15, D-97080 Würzburg, Federal Republic of Germany. 